\title{
Bertrand FAURÉ et Nicolas ARNAUD, $L a$ communication des organisations
}

Paris, La découverte 2014, 128 p. (collection Repères Gestion), ISBN

978-2-7071-8343-9-10€

James R. Taylor

\section{(2) OpenEdition}

\section{Journals}

Édition électronique

URL : http://journals.openedition.org/communicationorganisation/4838

DOI : 10.4000/communicationorganisation.4838

ISBN : 979-10-300-0155-6

ISSN : $1775-3546$

Éditeur

Presses universitaires de Bordeaux

\section{Édition imprimée}

Date de publication : 1 décembre 2014

Pagination : 301-304

ISBN : 978-2-86781-905-6

ISSN : $1168-5549$

\section{Référence électronique}

James R. Taylor, «Bertrand FAURÉ et Nicolas ARNAUD, La communication des organisations », Communication et organisation [En ligne], 46 | 2014, mis en ligne le 14 mars 2015, consulté le 22 septembre 2020. URL : http://journals.openedition.org/communicationorganisation/4838 ; DOI : https://doi.org/10.4000/communicationorganisation.4838 
Bertrand FAURÉ et Nicolas ARNAUD, La communication des organisations, Paris, La découverte 2014, 128 p. (collection Repères Gestion), ISBN 9782-7071-8343-9 - $10 €$

$\mathrm{Au}$ sein du champ de recherche sur l'organisation conçue comme un système de communication regroupant des communautés de pratique et des domaines de spécialisation différents mais complémentaires, un courant de recherche appelé dans la littérature anglo-saxonne "The Communicative Constitution of Organization » ou «CCO » (McPhee \& Zaug, 2000) est particulièrement vigoureux et novateur. Le texte de Fauré et Arnaud offre une 
introduction lucide et compréhensive aux principes de base de cette approche. Écrit dans un français élégant et expressif qui va à l'essentiel tout en suggérant beaucoup, il invite à porter un regard nouveau sur " la communication des organisations ».

Il est organisé en quatre chapitres: le premier introduit les concepts de base de cette théorie, soit le «comment » de la constitution de l'organisation dans, et par, l'interaction sociale, supportée et donnée sens, par le langage ; le deuxième chapitre se concentre sur la société des organisations ; le troisième explore les implications pour la gestion des entreprises, y compris l'impact des nouvelles technologies pour l'administration; le quatrième présente le défi méthodologique maintenant posé pour la recherche empirique qui se concentre sur le discours et les conversations des membres de l'organisation. Un sous-thème, aussi développé, est l'impact des nouvelles technologies de communication sur les structures de l'organisation et sa gestion.

Ce que ce livre souligne surtout est la logique de la constitution de l'organisation : son histoire et son exposition actuelle. La logique est celleci. Il faut que la communication engage les communicants : un engagement qui se fait par la communication, ainsi que les responsabilités mutuelles qui s'en découlent. C'est l'ensemble des engagements mutuels qui constituent les rapports sociaux qui, dans leur ensemble, forment ce que l'on pourrait appeler le tissu de l'organisation (Taylor, 1988). Comme les auteurs de ce livre l'écrivent, ceci amène le chercheur à focaliser sur le rôle du langage dans la composition des rapports sociaux, non seulement en formulant des idées, et donc une connaissance collective partagée typique des domaines spécialisés de toute sorte, mais en créant la responsabilité mutuelle des acteurs, y compris le pouvoir et la hiérarchie. L'étude du langage utilisé par les membres de l'organisation, en contexte, c'est-à-dire l'étude du discours, est devenue un domaine de recherche encore une fois de plus en plus international. Elle emprunte extensivement, à noter, une littérature écrite dans la langue française : celle de Saussure, Greimas, Ricoeur, etc.

Le concept d'organisation est ambigu (voir n'importe quel dictionnaire pour illustration) : elle est à la fois (1) un processus et (2) une unité, une " personne morale », reconnue comme telle dans la jurisprudence -c'est-àdire à la fois un ensemble d'acteurs et elle-même un acteur, dépendamment de la perspective adoptée. Le premier chapitre du livre de Fauré/Arnaud, explore l'histoire du relativement nouveau champ de recherche qui est connu en anglais, depuis son origine comme "organizational communication ", c'est-àdire l'organisation vue d'un angle particulier, celle de la communication. Cette spécialisation académique date des années 60 , la moitié d'un siècle. Parce que les concepts de communication à l'époque empruntaient pour la plupart aux méthodologies de la psychologie sociale et de la sociologie, l'emphase fut mise au départ sur la communication dans l'organisation, ce qui laissait croire à l'autonomie de l'organisation, existant avant la communication, mais qui 
après dépend de la communication, parce qu'elle a besoin de moyens pour réglementer les comportements de ses membres, individuels et groupes. La précédence a donc été accordée à l'organisation sur la communication. Vers la fin des années 80 plusieurs chercheurs, à commencer par ceux de l'université de Montréal, sont devenus insatisfaits par cette vue de l'organisation, car non seulement la communication est indispensable au fonctionnement de l'organisation mais parce que c'est logiquement la communication qui vient en premier. L'organisation n'est donc qu'une émanation de la communication. Sans communication, aucune organisation.

Si le premier chapitre met l'accent sur l'organisation comme ensemble d'acteurs le deuxième se concentre sur l'organisation comme elle-même un acteur social, c'est-à-dire un acteur inscrit dans les évolutions de la société... et y contribuant. Ce qui est novateur dans la discussion qui suit est le rôle organisant de la communication dans la construction d'une géographie de la vie des membres. L'organisation n'est pas qu'une composition d'acteurs et de tâches, elle est aussi une géographie turbulente d'espaces/temps. Selon la théorie relativiste d'Einstein et ses contemporains il n'existe pas un espace/ temps absolu et universel : tout dépend du point de vue. De même, les cadres spatio-temporels d'une communauté de praticiens ou de spécialistes sont des constructions vulnérables (Taylor \& Van Every, 1993) qui émanent de pratiques communicationnelles (le fond même de la théorie pragmatique de Charles Sanders Peirce au début du vingtième siècle). Ceci veut dire que la gestion des organisations équivaut à la tâche du géographe : écrire le plan de l'organisation. Nos propres recherches montrent qu'un des défis cruciaux pour l'administration est de réconcilier les écarts générés par la variation de pratiques localisées au regard d'une méta-conversation, typique de toute organisation complexe. Ce questionnement sur le rôle des dynamiques conversations-textes dans les organisations connaît actuellement une attention particulière de la part de plusieurs domaines de recherche (administrative science, organizational communication, discourse analysis, language at work...).

La première partie du livre offre un exposé clair et bien raisonné du développement historique de ce champ de recherche dans un domaine où n'existaient pour l'instant que des hand-books et récemment un manuel collectif franco-québécois (Grosjean \& Bonneville, 2010). Je le recommande à tout lecteur souhaitant découvrir les travaux de "L'école (du département de communication de l'université) de Montréal", travaux qui rassemblent aujourd'hui des chercheurs venus du monde entier (l'Europe, l'Amérique du nord et du sud, et la Pacifique (l'Australie, la Nouvelle Zélande et les îles des Philippines) lors de sessions prioritaires aux congrès annuels de l'International Communication Association (ou ICA) et du European Group of Organizational Studies (ou EGOS). En anglais on dirait qu'il s'agit d'un «state of the art».

Le troisième et quatrième chapitres présentent les défis méthodologiques que soulève une conception de l'organisation centrée sur la communication 
dans ses dimensions langagière et narrative et mettent l'accent sur les techniques de collecte et d'analyse des segments de discours que Fauré et Arnaud regroupent sous le terme d'" analyse de conversation ». J'ai moimême été inspiré, avec d'autres auteurs (Fauré, Brummans, Giroux \& Taylor, 2010 ; Taylor \& Van Every, 2011), par les recherches de Fauré sur les paroles comptables et la " performativité du langage des chiffres » (Fauré, 2013). Lui et Arnaud ont publié des travaux remarquables dans des revues prestigieuses en communication et gestion (Accounting, Organizations and Society, Governance, Organization and Management, Human Relations, Management Communication Quarterly). Ils savent de quoi ils parlent lorsqu'ils donnent des exemples d'analyse de discours mis au service d'une recherche sur la constitution de l'organisation par la communication.

Au final, ce livre « livre » ce qu'il promet : une introduction lucide, animée et bien informée concernant les origines, le développement et les avancées récentes d'un champ de recherche de plus en plus international et prioritaire. C'est un livre important pour comprendre les organisations, comment elles existent et ce qu'elles font exister.

Jomes R. Toụlor ${ }^{4}$

4 James R. (Jim) Taylor est Professeur Emérite de Communication á l'Université de Montréal et professeur fondateur du Département de Communication à cette université au début des 1970s. Il s'est spécialisé au champ de recherché connu comme l'Organizational Communication. Sa recherche et son enseignement a surtout focalizé sur la capacité des gens, en mobilisant le texte et la conversation, de configurer des réseaux de rapports et, en se faisant, de construire la personne de l'organisation et ainsi une entité qui les englobe.

Son dernier livre, en collaboration avec Elizabeth Van Every, est « When organization fails: Why authority matters » (Routledge). http://www.taylorvanevery.com/www.taylorvanevery.com/Main.html 